\title{
Creation and characterisation of $11 \beta$ hydroxysteroid dehydrogenase type 1 transgenic mesenchymal stem cells for use in models of acute respiratory distress syndrome
}

RAHUL Y. MAHIDA ( $\nabla$ r.mahida@bham.ac.uk)

University of Birmingham College of Medical and Dental Sciences https://orcid.org/0000-0003-11008684

Zhengqiang Yuan

University College London

Krishna K. Kolluri

University College London

Sebastian T. Lugg

University of Birmingham College of Medical and Dental Sciences

Aaron Scott

University of Birmingham College of Medical and Dental Sciences

Dhruv Parekh

University of Birmingham College of Medical and Dental Sciences

Rowan S. Hardy

University of Birmingham College of Medical and Dental Sciences

Gareth G. Lavery

University of Birmingham College of Medical and Dental Sciences

Sam M. Janes

University College London

David R. Thickett

University of Birmingham College of Medical and Dental Sciences

Research article

Keywords: MSCs, HSD-1, Transfection, Lentivirus, ARDS

Posted Date: September 25th, 2019

DOI: https://doi.org/10.21203/rs.2.14766/v1 
License: (c) (i) This work is licensed under a Creative Commons Attribution 4.0 International License. Read Full License 


\section{Abstract}

Background: Human bone marrow mesenchymal stem cell (MSC) administration reduces inflammation in pre-clinical models of lung injury, however clinical efficacy in patients with acute respiratory distress syndrome (ARDS) has not been shown. Upregulation of the glucocorticoid activating enzyme, $11 \beta$ hydroxysteroid dehydrogenase type 1 (HSD-1) within the alveolar space elevates local anti-inflammatory cortisol levels, and promotes alveolar macrophage efferocytosis. Administration of HSD-1 transgenic MSCs (tMSCs) that overexpress HSD-1 may enhance local cortisol activation. This combined cellular and gene therapy may be more effective at reducing inflammation in ARDS than cellular therapy alone.

Methods: Molecular cloning was used to create a recombinant lentiviral vector containing the HSD-1 and green fluorescent protein (GFP) transgenes. MSCs were transfected using this lentivirus, then transfection efficiency was assessed using flow-cytometry. HSD-1 transgene expression was assessed using immunofluorescence and western blotting. Thin layer chromatography was used to assess HSD-1 function in tMSCs. Bi-lineage differentiation and flow-cytometry were used to determine whether tMSCs maintained a stem cell phenotype.

Results: A recombinant lentiviral vector was created containing the HSD-1 and GFP transgenes under the control of a tetracycline promoter. MSCs were successfully transfected, with a transfection efficiency of $91.1 \%$. HSD-1 transgene expression was confirmed by immunofluorescence and western blot. Functional HSD-1 activity was evident within tMSCs, with predominant reductase cortisol activation, following treatment with the transcriptional activator doxycycline. HSD-1 reductase activity was maintained for 72 hours after doxycycline was removed from tMSCs. HSD-1 tMSCs maintained the capacity for osteogenic and adipogenic differentiation, and maintained expression of MSC surface markers.

Conclusions: We successfully transduced tMSCs to express the HSD-1 transgene that functions predominantly as a reductase. Administration of HSD-1 tMSCs to in vivo models of ARDS may attenuate inflammation through activation of cortisol in the alveolar space.

\section{Background}

Human bone marrow mesenchymal stem cells (MSCs) are pluripotent, retaining the ability to differentiate into several distinct cell types. Numerous pre-clinical studies have shown that administration of MSCs can attenuate inflammation and promote repair in models of lung injury. MSCs have been shown to be efficacious in small and large animal models of lung injury(1-4), and in both animal and human injured ex-vivo lung perfusion models(5-8). Phase 1 and 2a clinical trials in patients with Acute Respiratory Distress Syndrome (ARDS) have shown that MSC administration is safe, feasible and well-tolerated, with no adverse effects(9-11). Phase 3 clinical trials are still required to determine MSC efficacy in ARDS patients. Allogenic MSCs can be administered without inducing an immune response(12). MSCs can be expanded in vitro, retain their efficacy following cryopreservation $(13,14)$, and localise to the lungs following intravenous administration $(15,16)$. These factors support the use of MSCs as a clinical therapy 
for ARDS. The two main mechanisms by which MSC attenuate lung injury are the promotion of epithelial and endothelial repair, and modulation of immune function to increase bacterial clearance and aid resolution of inflammation(17). These mechanisms can be initiated by either cell-to-cell contact or release of paracrine factors. However, studies have also shown that MSCs lose their intrinsic anti-inflammatory capabilities with repeated in vitro passage(18). Therefore, the expansion required to generate sufficient MSCs for clinical therapy may also decrease their anti-inflammatory functions, thereby limiting their therapeutic potential.

The characteristics of MSCs which support their use as clinical therapy also make them ideal vectors for gene therapy. Use of transgenic MSCs (tMSCs) in murine models of lung injury have shown that the synergistic anti-inflammatory action of combined cellular and gene therapy can be superior to that of cellular therapy alone(19-21). Transgenes delivered by tMSCs in lung injury models have included Interleukin-10(19) and Angiopoetin-1(20,21). MSCs have also been considered as vectors for gene therapy in lung cancer with. The authors previously created tMSCs expressing TNF-related apoptosisinducing ligand (TRAIL), using a lentiviral vector containing the Tet-on system were utilised(22, 23). The TRAIL transgene was under the control of a tetracycline promoter and only expressed in the presence of doxycycline. In this way, the Tet-on system allowed efficient control of TRAIL transgene expression. It was shown that TRAIL tMSCs can kill cancer cells in murine metastatic lung cancer and malignant mesothelioma models following intravenous administration(22, 24).

In vivo, the enzyme $11 \beta$ Hydroxysteroid Dehydrogenase Type-1 (HSD-1) acts as a reductase, converting the inactive glucocorticoid cortisone to its active counterpart, cortisol and locally amplifying glucocorticoid action within tissues(25). Previous studies have demonstrated that elevated HSD-1 and local cortisol activation promote anti-inflammatory macrophage polarisation, efferocytosis and resolution of inflammation(26-28). We postulated that administration of tMSCs expressing the HSD-1 transgene in murine models of lung injury would elevate alveolar cortisol levels, and in combination with the innate immuno-modulatory abilities of the MSCs would act synergistically to enhance alveolar macrophage efferocytosis and attenuate inflammation, and support resolution in ARDS.

In this study we describe the methodology and validation for the creation of functional HSD-1 expressing tMSCs, using a lentiviral vector containing the Tet-on system. We also show that the HSD-1 tMSCs maintain an MSC phenotype following transfection.

\section{Methods}

\section{Creation of recombinant HSD-1 lentiviral plasmid}

A lentiviral plasmid (pRRL-cPPT-hPGK-mcs-WPRE) into which the Tet-on system elements have been introduced previously, had the reporter gene MuSEAP excised and replaced with the IRES-eGFP sequence (Internal Ribosome Entry Site-Enhanced Green Fluorescent Protein from pENTR1A) by Professor Sam 
Janes' group at UCL(22). This modified lentiviral plasmid was used as the backbone for the incorporation of human $11 \beta$ Hydroxysteroid Dehydrogenase Type-1 (HSD-1) DNA.

Human HSD-1 cDNA (amino acids 1-293, Origene) was amplified and restriction sites for Mlul and BstB1 were introduced using polymerase chain reaction (PCR). Forward amplification primer sequence (Invitrogen) was CGTACGCGTGCCACCATGGCTTTTATGAAAAAATATCTCCTCCC. Reverse amplification primer sequence was GTCGTTCGAACTACTTGTTTATGAATCTGTCC. The HSD-1 PCR product and the lentiviral backbone plasmid were then both sequentially digested with Mlul then BstB1 (New England Biolabs). Digestion of the lentiviral backbone plasmid created a break between the Tet-on promoter and the IRES-eGFP. The digested HSD-1 insert was ligated into the digested lentivirus plasmid vector next to the IRES-eGFP, using T4 DNA ligase (New England Biolabs) to create the recombinant plasmid.

Recombinant plasmids were amplified in DH5a Escherichia coli (E. coli, ThermoFisher Scientific) grown in LB broth supplemented with $100 \mu \mathrm{g} / \mathrm{ml}$ ampicillin, and purified using HiSpeed Plasmid Maxip kits (Qiagen). Recombinant plasmid constructs were confirmed by DNA sequence analysis (Source Bioscience).

\section{Production of Recombinant HSD-1 Lentivirus}

Human Embryonic Kidney 293T cells (HEK 293T, Sigma) were cultured in T175 flasks (ThermoFisher Scientific) with Dulbecco's Modified Eagle's Medium (DMEM, Sigma) including 10\% Foetal Bovine Serum (FBS), $100 \mathrm{U} / \mathrm{mL}$ penicillin, $100 \mathrm{ug} / \mathrm{mL}$ streptomycin and $2 \mathrm{mM} \mathrm{L}$-glutamine (all from Sigma) at $37^{\circ} \mathrm{C}$ and $5 \% \mathrm{CO}_{2}$ until reaching $80-90 \%$ confluence. HEK 293T cells were then transfected using a secondgeneration packaging system. Culture medium was removed from flasks of HEK 293T cells, and replaced with $13 \mathrm{mls}$ of DMEM / $10 \%$ FBS and $2 \mathrm{mls}$ of a $150 \mathrm{nM} \mathrm{NaCl}$ solution containing $80 \mu$ linear polyethylenimine (jetPEI transfection reagent, Polyplus transfection), $20 \mu \mathrm{g}$ recombinant HSD-1 plasmid, $13 \mu \mathrm{g}$ packaging plasmid pCMV-dR8.74, and $7 \mu \mathrm{g}$ envelope plasmid pMD.G2. Media was changed for $15 \mathrm{ml}$ fresh DMEM / 10\% FBS at 4 hours and 24 hours post-transfection. At 48 hours post-transfection, media containing recombinant lentivirus was collected and fresh media added; media was again collected at 72 hours post-transfection. Supernatant media was centrifuged at $300 \mathrm{~g}$ for 10 minutes at $4^{\circ} \mathrm{C}$ (Eppendorf AG 5810R) to pellet any cellular debris, then passed through a $0.45 \mu \mathrm{m}$ filter (Sartorius). Recombinant lentivirus within the media was then concentrated by ultracentrifugation (SW28 rotor, Optima LE80K Ultracentrifuge, Beckman) at $52,000 \mathrm{~g}$ and $4^{\circ} \mathrm{C}$ for 2 hours. Supernatants were completely discarded and the lentiviral pellet re-suspended in serum-free DMEM, prior to storage at $-80^{\circ} \mathrm{C}$.

\section{Determination of Viral Titre}

HEK 293 T cells were plated at 50,000 per well in a 12-well plate and incubated in DMEM / 10\% FBS at $37^{\circ} \mathrm{C}$ and $5 \% \mathrm{CO}_{2}$ overnight. Wells were treated with $8 \mu \mathrm{g} / \mathrm{ml}$ hexamethadine bromide (Polybrene, Sigma) transfection reagent, and serial dilutions of recombinant HSD-1 lentivirus (1:100 to 1:10,000). Two control wells received polybrene but no lentivirus. After 4 hours incubation, media was changed to DMEM 
/ 10\% FBS. After a further 24 hours media was changed again and $10 \mu \mathrm{g} / \mathrm{ml}$ doxycycline was added, which acted as the transcriptional activator for the HSD-1 and GFP transgenes. After a further 24 hours, cells were harvested and assessing for GFP expression using flow cytometry (LSR Fortessa X-20, BD Biosciences). The viral titre was calculated(29) as follows: $(P \times N) / V . P=$ Proportion of GFP positive cells, $\mathrm{N}=$ Number of HEK 293T cells, $\mathrm{V}=$ Volume of virus in $\mathrm{ml}$.

\section{Transfection of MSCs}

Primary human MSCs were cultured in a-Minimal Essential Media (aMEM, ThermoFisher) containing 16\% FBS. Prior to and following transfection, these MSCs were cultured using tetracycline-free FBS (lot 42G9273K, ThermoFisher Scientific), to prevent uncontrolled expression of GFP and HSD-1 transgenes. MSCs from a single donor at passage 2 were plated at 100,000 per T75 flask and cultured overnight at $37^{\circ} \mathrm{C}$ and $5 \% \mathrm{CO}_{2}$ to allow adherence. Media was removed from each flask and replaced with $5 \mathrm{mls}$ aMEM containing $8 \mu \mathrm{g} / \mathrm{ml}$ polybrene and 200,000 lentiviral transduction units. MSCs were transfected with a multiplicity of infection (MOI) of 2 virus particles for each cell. Doxycycline acted as a transcriptional activator for both the HSD-1 and GFP transgenes, which were under the control of a tetracyclinedependant promoter.

\section{Assessment of HSD-1 transgene expression by Flow Cytometry and Immunofluorescence}

Flow cytometry and immunofluorescence (IF) were used to determine the percentage of transgeneactivated transgenic MSCs (tMSCs) expressing the HSD-1 protein following intracellular staining. The tMSCs were cultured with $10 \mu \mathrm{g} / \mathrm{ml}$ doxycycline for 48 hours, before being fixed in $4 \%$ paraformaldehyde, then made permeable by incubating in saponin buffer (PBS / 10\% FBS / $0.1 \%$ saponin). Cells were incubated with primary rabbit anti-human HSD-1 monoclonal antibody (ab157223, Abcam) at 1:100 dilution, then secondary donkey anti-rabbit IgG H\&L antibody Alexa-fluor ${ }^{\circledR} 555$ (ab150074, Abcam) at 1:500 dilution, prior to analysis on flow cytometry (LSR Fortessa X-20, Becton-Dickinson). For IF, steps were followed as above within chamber-well slides (Nunc, Lab-Tek, ThermoFisher Scientific), following which mounting buffer containing 4',6-diamidino-2-phenylindole (DAPI Prolong Gold Antifade Reagent, ThermoFisher Scientific) was added. Coverslips were placed on the slides and images taken with a fluorescent microscope (Axioskop 2, Carl Zeiss Ltd., UK).

\section{Western Blot assessment of HSD-1 Transgene Expression}

Expression of HSD-1 protein in tMSCs was assessed by Western Blot. $1 \times 10^{6}$ non-transfected MSCs and tMSCs (previously cultured with $10 \mu \mathrm{g} / \mathrm{ml}$ doxycycline) were harvested. Cells were lysed using RIPA buffer (Cell Signalling) then protein concentrations of cell lysates were calculated using a bicinchoninic acid assay (ThermoFisher Scientific). Samples were denatured at $70^{\circ} \mathrm{C}$ and resolved on a Bolt $4-12 \%$ Bis-Tris- 
Plus gel (ThermoFisher) and MES SDS (2-(N-morpholino)ethanesulfonic acid, sodium dodecyl sulfate, ThermoFisher) running buffer at $15 \mu \mathrm{g}$ or $22 \mu \mathrm{g}$ protein/well. Samples were then transferred onto a membrane using Novex iBlot transfer system (ThermoFisher). The membrane was incubated with primary rabbit anti-HSD-1 monoclonal antibody (ab157223, Abcam) at 1:10,000 dilution, then Goat polyclonal anti-Rabbit HRP-conjugated antibody (P0448, Dako) at 1:2000 dilution, then Horseradish Peroxidase substrate (HRP substrate, Merck Millipore) prior to detection (Image Quant LAS 4000, GE). To assess total protein loading the membrane was stripped, labelled with anti- $\beta$-tubulin rabbit antibody (9F3, Cell Signalling) at 1:1000, then Goat anti-Rabbit HRP-conjugated antibody at 1:2000, then HRP substrate prior to detection.

\section{Adipogenic and osteogenic differentiation}

To determine if tMSCs retained typical stem cell plasticity or if transfection induced terminal differentiation, bi-lineage adipogenic and osteogenic differentiation kits (StemPro) were used; protocols were as per manufacturer's guidance. MCSs cultured in aMEM / 16\% FBS acted as negative control. For adipocyte detection, MSCs were stained with a $0.2 \%$ stock solution of Oil Red O (Sigma-Aldrich) which is taken up by lipid inclusion vacuoles. For osteocyte detection, MSCs were stained with a $2 \%$ solution of Alizarin Red S (Sigma-Aldrich) which is taken up by calcium deposits. Following staining, photographs were taken via a microscope (Zeiss AxioVert A1).

\section{Flow cytometry assessment of mesenchymal stem cell surface markers}

HSD-1 tMSCs and non-transgenic MSCs were labelled with the following anti-human antibodies or their isotype controls: CD105-BV786, CD73-BV421, CD90-PE (positive cocktail, all purchased from BD Biosciences) or CD14-APC, CD19-APC, CD34-APC, CD45-APC, and HLADR-APC (negative cocktail, all purchased from ThermoFisher Scientific). Surface marker expression was assessed by flow cytometry (LSR Fortessa X-20, BD Biosciences). Compensation and analysis was performed using FACSDiva Software (BD Biosciences).

\section{HSD-1 Functional Assays}

Steroid metabolism of cortisol and cortisone in tMSC was determined using tritiated steroids (Perkin Elmer, Beaconsfield, Buckinghamshire, UK) and scanning thin layer chromatography as previously described $(30,31)$. Briefly, non-transfected MSCs were cultured in either aMEM / 16\% FBS. Cultures were pre-treated with either vehicle control, $10 \mu \mathrm{g} / \mathrm{ml}$ doxycycline, $50 \mathrm{ng} / \mathrm{ml}$ tumour necrosis factor-a (TNF-a, Peprotech) or $100 \mathrm{ng} / \mathrm{ml}$ Lipopolysaccaride (LPS, Sigma) for 48 hours. To inhibit HSD-1 activity, cells were incubated with $10^{-7} \mathrm{M}$ glycyrrhetinic acid (Sigma) for 24 hours prior to functional assays being performed. Steroids were extracted in dichloromethane and separated by thin layer chromatography on 
silicate plates. Thin-layer chromatography plates were analysed by Bioscan imager (Bioscan, Washington, DC, USA) to detect the formation of ${ }^{3} \mathrm{H}$-corticosterone. The fractional conversion of steroids was calculated as pmol product/per $\mathrm{mg}$ protein/hr.

\section{Statistical Analysis}

Data was analysed using Prism 8 software (GraphPad). Normality of data was assessed using the D’Agostino \& Pearson test. Differences between continuously distributed non-parametric data were assessed using Mann-Whitney tests. Differences between three or more non-parametric data sets were assessed using the Kruskal-Wallis one-way analysis of variance (ANOVA) and Dunn's multiple comparison tests. Two-tailed $p$-values of $<0.05$ were considered as significant. Results from nonparametric data are shown as median and interquartile range.

\section{Results}

\section{Creation of HSD-1 Transgenic Mesenchymal Stem Cells}

Human bone marrow MSCs were transfected with recombinant HSD-1 lentivirus, using a multiplicity of infection (MOI) of 2. Following transfection and culture with $10 \mu \mathrm{g} / \mathrm{ml}$ doxycycline, tMSCs were visualised under fluorescence microscopy to assess for GFP expression (Figure 1A-D). We next confirmed that transgene-activated tMSCs expressed the HSD-1 protein. Transgene-activated tMSCs were stained intracellularly for HSD -1 to assess for transfection efficiency, which was found to be $90.1 \%$ (SD $2.6 \%, n=$

3 ) on flow cytometry (Figure 2A-B). Both immunofluorescence and western blot experiments (Figure 2C-G) found that HSD-1 protein was present in transgene-active tMSCs, and absent in non-transfected MSCs. Immunofluorescence also showed that HSD-1 protein localised to the cytoplasm of tMSCs. These results indicated that the transfection process had been successful and that tMSCs were able to produce the HSD-1 protein when cultured with doxycycline (transcriptional activator). These experiments also confirmed that there was no expression of HSD-1 in non-transfected MSCs.

\section{Transgenic MSCs express functional HSD-1}

We showed that the HSD-1 expressed within tMSCs was functional as a reductase, able to convert cortisone into cortisol. tMSCs were treated with doxycycline for 72hrs to induce HSD1 transgene expression, prior to measuring HSD-1 activity. Duration of doxycycline treatment significantly affects functional HSD-1 reductase activity in tMSCs (figure 3A). HSD-1 activity in tMSCs peaks after 48hrs exposure to doxycycline (median $8.23 \mathrm{pM} /$ hour/100,000 cells). HSD-1 reductase activity was also seen in doxycycline-naïve tMSCs (cultured in media containing tetracycline-free FBS); however this was significantly lower than reductase activity following doxycycline treatment for 48 hours or 72 hours (figure $3 \mathrm{~A}$ ). We then showed tMSCs maintain a constant level of functional HSD-1 reductase activity up to 72 hours after doxycycline removal (figure 3B). 
Doxycycline-naïve tMSCs had undetectable expression of the GFP transgene (co-expressed with the HSD-1 transgene), as assessed by fluorescent microscopy and flow cytometry (data not shown). Treatment of doxycycline-naïve tMSCs with glycyrrhetinic acid (GA), which inhibits HSD-1 activity, reductase activity significantly diminished (figure $3 \mathrm{C}$ ), indicating that the reductase activity we observed was due to HSD-1 activity.

Non-transfected MSCs were also tested for HSD-1 activity: Untreated, with doxycycline, or with inflammatory mediators (LPS and TNFa for 24 hours) to mimic an inflammatory environment prior to use in HSD-1 functional assays. None of these conditions resulted in detectable HSD-1 reductase activity (figure 3D).

\section{HSD-1 Transgenic MSCs maintain an MSC phenotype}

To show that HSD-1 tMSCs maintain a stem cell phenotype, we assessed differentiation capacity and surface marker expression as per the International Society for Cellular Therapy (ISCT) criteria(32). Adipogenic and osteogenic differentiation experiments showed that tMSCs retain the capacity to differentiate into adipogenic and osteogenic lines, to the same degree as non-transfected MSCs (figure $4 A-C)$.. The tMSCs also adhere to plastic surfaces equally as well as non-transfected MSCs under control culture conditions, which is another defining criteria of an MSCs according to the ISCT.

Phenotypic assessment of HSD-1 tMSCs by flow cytometry revealed tMSCs expressed positive markers CD90, CD105 and CD73 (Figure 4D-G). HSD-1 tMSCs also lacked expression of negative markers CD14, CD19, CD34, CD45 and HLA-DR. Therefore, HSD-1 tMSCs retain the MSC surface marker configuration as per ISCT criteria. These results indicate that tMSCs retain an MSC phenotype and differentiation capabilities following transfection with recombinant HSD-1 lentivirus.

\section{Discussion}

In this study we generated and validated a recombinant lentivirus containing both the HSD-1 and GFP transgenes under the control of a tetracycline promoter. In subsequent transfection experiments we generated human MSCs containing this recombinant lentivirus to create HSD-1 tMSCs, with a transfection efficiency of $90.1 \%$. Following exposure to doxycycline to induce transgene expression, the tMSCs co-expressed both HSD-1 and GFP protein with functional activity experiments confirming reductase activity and cortisol activation. The induction of HSD1 reductase activity peaked at 48 hours following culture with doxycycline (concentration). This identified the minimum time required for incubation with doxycycline before maximal HSD-1 reductase activity is seen, and guided the preparation of tMSCs for future experiments. Comparing with unpublished human alveolar macrophage HSD-1 activity data from our group, we found that the transgene-active tMSCs exhibit 41 times greater HSD-1 reductase activity than normal alveolar macrophages (8.23 vs $0.2 \mathrm{pM} /$ hour/100,000 cells). Therefore, we predict that a relatively low dose of tMSCs would be able to significantly enhance alveolar cortisol levels in models of ARDS. 
Use of doxycycline in co-culture experiments and models of lung injury would have a significant confounding factor due to its action as an antibiotic and matrix metalloproteinase inhibitor. As such, maintenance of maximal HSD-1 activity after doxycycline is removed from tMSC culture is a key factor. We found that after 48 hours culture with doxycycline, the tMSCs maintain constant levels of HSD-1 reductase activity for at least a further 72 hours after removal of doxycycline is removed from culture media. Therefore, tMSCs pre-treated with doxycycline for 48hours, will achieve and maintain maximal HSD-1 activity in the absence of doxycycline for the duration of co-culture experiments or murine models of lung injury, mitigating a potential confounding factor for this system.

The HSD-1 functional assays demonstrated that non-transfected MSCs do not express functional HSD1. They have also shown that MSCs do not express functional HSD-1 in response to doxycycline, LPS or TNF-a treatment. This is important to clarify, as any functional HSD-1 activity seen in doxycyclinetreated tMSCs is therefore due to expression of the HSD-1 transgene, and not due to upregulation of the HSD-1 gene from the native MSC DNA. Future experimental applications of these tMSCs will include coculture experiments with ARDS alveolar macrophages, and investigating efficacy in murine models of lung injury. These models will both contain high levels of pro-inflammatory mediators in the alveolar space, including LPS and TNF-a. Therefore, the HSD-1 activity seen in tMSCs exposed to an inflammatory environment will only be due to expression of the HDS-1 transgene.

Unexpectedly, HSD-1 reductase activity was observed in doxycycline-naïve tMSCs which had been cultured in media containing tetracycline-free FBS. Doxycycline-naïve tMSCs had undetectable expression of the GFP transgene, which is co-expressed with the HSD-1 transgene (as assessed by flow cytometry and fluorescent imaging). After treating doxycycline-naïve tMSCs with glycyrrhetinic acid (GA) which inhibits HSD-1 activity, the reductase activity significantly diminished, so that the observed reductase activity was due to HSD-1 expression. We postulate there remains a small but detectable transgene expression in the absence of the transcriptional activator. In the case of the GFP transgene, this protein expression was so minimal as not to be detected on fluorescent microscopy and flow cytometry. However, it appeared that even this minimal expression of HSD-1 protein could result in detectable enzymatic activity; the degree of HSD-1 protein expression is not directly proportional to the functional activity observed. This effect was not seen with doxycycline-naïve TRAIL tMSCs(22), because TRAIL is not an enzyme and therefore a minimal amount of protein expression would give an equally minimal effect. We therefore planned to pre-treat doxycycline-naive tMSCs with GA to minimise the underlying HSD-1 activity as much as possible prior to use in transwell co-culture and murine experiments.

We showed that the tMSCs retain a stem cell phenotype and possess the associated multipotency; the transfection process had not caused differentiation of the MSCs. Therefore, by retaining an MSC phenotype, the retained intrinsic anti-inflammatory capabilities of the tMSCs could combine synergistically with elevated HSD-1 transgene expression to enhance alveolar macrophage function and reduce inflammation. 
In summary, we have successfully created tMSCs which express functional HSD-1 enzyme, which is able to local activate cortisol from the inactive precursor cortisone. Future studies will focus on characterising the efficacy of tMSCs in murine models of lung injury and in vitro transwell co-culture experiments with alveolar macrophages from ARDS patients, to determine if this therapeutic strategy can attenuate inflammation in ARDS.

\section{Abbreviations}

$\mathrm{APC}=$ Allophycocyanin. ARDS $=$ Acute respiratory distress syndrome. aMEM $=$ a-Minimal essential media. ANOVA = Analysis of variance. DAPI = 4',6-diamidino-2-phenylindole. DMEM = Dulbecco's Modified Eagle's Medium. FBS = Foetal bovine serum. GA = glycyrrhetinic acid. GFP = Green fluorescent protein. $\mathrm{HEK}=$ Human Embryonic Kidney cell. HSD-1 = $11 \beta$ hydroxysteroid dehydrogenase type-1. ISCT $=$ International society for cellular therapy. LPS = Lipopolysaccaride. $\mathrm{MOI}=$ multiplicity of infection. (t)MSC $=($ Transgenic) Mesenchymal stem cell. $\mathrm{PCR}=$ polymerase chain reaction. $\mathrm{SD}=$ Standard deviation. TNFa $=$ Tumour necrosis factor a. TRAIL = TNF-related apoptosis-inducing ligand. UCL = University College London.

\section{Declarations}

\section{Ethics approval and consent to participate}

Bone marrow MSCs were obtained from human donors who had given informed written consent at the Institute of Regenerative Medicine at Texas A\&M, USA.

\section{Consent for publication}

Not applicable

\section{Availability of data and material}

Data and material are available on reasonable request to the corresponding author.

\section{Competing interests:}

The authors declare they have no competing interests.

\section{Funding:}

This study was funded by grants MR/N021185/1 and MR/L002736/1 from the Medical Research Council, U. K. 


\section{Authors' contributions:}

RYM, ZY and KK performed the experiments. RH and GL provided advice and guidance for HSD-1 functional assays. STL, DP and AS assisted with HSD -1 data analysis. RYM, AS and DRT wrote the manuscript. SMJ, DRT, RH and GL supervised the work and reviewed the manuscript. All authors read and approved the final manuscript.

\section{Acknowledgements}

We would like to thank the late Iwona Bujalska for her assistance with the HSD-1 functional assays described here.

\section{References}

1.Cárdenes N, Cáceres E, Romagnoli M, Rojas M. Mesenchymal Stem Cells: A Promising Therapy for the Acute Respiratory Distress Syndrome. Respiration. 2013;85(4):267-78.

2.McIntyre LA, Moher D, Fergusson DA, Sullivan KJ, Mei SH, Lalu M, et al. Efficacy of Mesenchymal Stromal Cell Therapy for Acute Lung Injury in Preclinical Animal Models: A Systematic Review. PLoS One. 2016;11(1):e0147170.

3.Asmussen S, Ito H, Traber DL, Lee JW, Cox RA, Hawkins HK, et al. Human mesenchymal stem cells reduce the severity of acute lung injury in a sheep model of bacterial pneumonia. Thorax. 2014;69(9):819-25.

4.Rojas M, Cardenes N, Kocyildirim E, Tedrow JR, Caceres E, Deans R, et al. Human adult bone marrowderived stem cells decrease severity of lipopolysaccharide-induced acute respiratory distress syndrome in sheep. Stem cell research \& therapy. 2014;5(2):42.

5.Lee JW, Fang X, Gupta N, Serikov V, Matthay MA. Allogeneic human mesenchymal stem cells for treatment of E. coli endotoxin-induced acute lung injury in the ex vivo perfused human lung. Proceedings of the National Academy of Sciences. 2009;106(38):16357-62.

6.Lee JW, Krasnodembskaya A, McKenna DH, Song Y, Abbott J, Matthay MA. Therapeutic effects of human mesenchymal stem cells in ex vivo human lungs injured with live bacteria. American journal of respiratory and critical care medicine. 2013;187(7):751-60.

7.McAuley DF, Curley GF, Hamid UI, Laffey JG, Abbott J, McKenna DH, et al. Clinical grade allogeneic human mesenchymal stem cells restore alveolar fluid clearance in human lungs rejected for transplantation. American journal of physiology Lung cellular and molecular physiology. 2014;306(9):L809-15. 
8. Rojas M, Parker RE, Thorn N, Corredor C, lyer SS, Bueno M, et al. Infusion of freshly isolated autologous bone marrow derived mononuclear cells prevents endotoxin-induced lung injury in an ex-vivo perfused swine model. Stem cell research \& therapy. 2013;4(2):26.

9.Zheng G, Huang L, Tong H, Shu Q, Hu Y, Ge M, et al. Treatment of acute respiratory distress syndrome with allogeneic adipose-derived mesenchymal stem cells: a randomized, placebo-controlled pilot study. Respiratory research. 2014;15:39.

10.Wilson JG, Liu KD, Zhuo H, Caballero L, McMillan M, Fang X, et al. Mesenchymal stem (stromal) cells for treatment of ARDS: a phase 1 clinical trial. The Lancet Respiratory Medicine. 2015;3(1):24-32.

11. Matthay MA, Calfee CS, Zhuo H, Thompson BT, Wilson JG, Levitt JE, et al. Treatment with allogeneic mesenchymal stromal cells for moderate to severe acute respiratory distress syndrome (START study): a randomised phase 2a safety trial. The Lancet Respiratory medicine. 2019;7(2):154-62.

12.Patel SA, Sherman L, Munoz J, Rameshwar P. Immunological properties of mesenchymal stem cells and clinical implications. Archivum immunologiae et therapiae experimentalis. 2008;56(1):1-8.

13.Devaney J, Horie S, Masterson C, Elliman S, Barry F, O'Brien T, et al. Human mesenchymal stromal cells decrease the severity of acute lung injury induced by E. coli in the rat. Thorax. 2015;70(7):625-35.

14.Yuan Z, Lourenco Sda S, Sage EK, Kolluri KK, Lowdell MW, Janes SM. Cryopreservation of human mesenchymal stromal cells expressing TRAIL for human anti-cancer therapy. Cytotherapy. 2016;18(7):860-9.

15.Nemeth K, Leelahavanichkul A, Yuen PST, Mayer B, Parmelee A, Doi K, et al. Bone marrow stromal cells attenuate sepsis via prostaglandin E2-dependent reprogramming of host macrophages to increase their interleukin-10 production. Nat Med. 2009;15(1):42-9.

16.Rojas M, Xu J, Woods CR, Mora AL, Spears W, Roman J, et al. Bone Marrow-Derived Mesenchymal Stem Cells in Repair of the Injured Lung. American journal of respiratory cell and molecular biology. 2005;33(2):145-52.

17.Laffey JG, Matthay MA. Fifty Years of Research in ARDS. Cell-based Therapy for Acute Respiratory Distress Syndrome. Biology and Potential Therapeutic Value. American journal of respiratory and critical care medicine. 2017;196(3):266-73.

18.Choi MR, Kim HY, Park J-Y, Lee TY, Baik CS, Chai YG, et al. Selection of optimal passage of bone marrow-derived mesenchymal stem cells for stem cell therapy in patients with amyotrophic lateral sclerosis. Neuroscience Letters. 2010;472(2):94-8.

19.Wang C, Lv D, Zhang X, Ni ZA, Sun X, Zhu C. Interleukin-10-Overexpressing Mesenchymal Stromal Cells Induce a Series of Regulatory Effects in the Inflammatory System and Promote the Survival of Endotoxin-Induced Acute Lung Injury in Mice Model. DNA and cell biology. 2018;37(1):53-61. 
20.Mei SHJ, McCarter SD, Deng Y, Parker CH, Liles WC, Stewart DJ. Prevention of LPS-Induced Acute Lung Injury in Mice by Mesenchymal Stem Cells Overexpressing Angiopoietin 1. PLoS Med. 2007;4(9):e269.

21.Xu J, Qu J, Cao L, Sai Y, Chen C, He L, et al. Mesenchymal stem cell-based angiopoietin-1 gene therapy for acute lung injury induced by lipopolysaccharide in mice. The Journal of pathology. 2008;214(4):472-81.

22.Loebinger MR, Eddaoudi A, Davies D, Janes SM. Mesenchymal stem cell delivery of TRAlL can eliminate metastatic cancer. Cancer research. 2009;69(10):4134-42.

23.Barde I, Zanta-Boussif MA, Paisant S, Leboeuf M, Rameau P, Delenda C, et al. Efficient control of gene expression in the hematopoietic system using a single Tet-on inducible lentiviral vector. Mol Ther. 2006;13(2):382-90.

24.Sage EK, Kolluri KK, McNulty K, Lourenco Sda S, Kalber TL, Ordidge KL, et al. Systemic but not topical TRAIL-expressing mesenchymal stem cells reduce tumour growth in malignant mesothelioma. Thorax. 2014;69(7):638-47.

25.Tomlinson JW, Stewart PM. Cortisol metabolism and the role of $11 \beta$-hydroxysteroid dehydrogenase. Best Practice \& Research Clinical Endocrinology \& Metabolism. 2001;15(1):61-78.

26.Gilmour JS, Coutinho AE, Cailhier JF, Man TY, Clay M, Thomas G, et al. Local amplification of glucocorticoids by 11 beta-hydroxysteroid dehydrogenase type 1 promotes macrophage phagocytosis of apoptotic leukocytes. Journal of immunology. 2006;176(12):7605-11.

27.Liu Y, Cousin JM, Hughes J, Van Damme J, Seckl JR, Haslett C, et al. Glucocorticoids promote nonphlogistic phagocytosis of apoptotic leukocytes. Journal of immunology. 1999;162(6):3639-46.

28.Hardy RS, Fenton C, Croft AP, Naylor AJ, Begum R, Desanti G, et al. 11 Beta-hydroxysteroid dehydrogenase type 1 regulates synovitis, joint destruction, and systemic bone loss in chronic polyarthritis. Journal of autoimmunity. 2018;92:104-13.

29.Giry-Laterriere M, Verhoeyen E, Salmon P. Lentiviral vectors. Methods in molecular biology (Clifton, NJ). 2011;737:183-209.

30.Bujalska IJ, Kumar S, Hewison M, Stewart PM. Differentiation of adipose stromal cells: the roles of glucocorticoids and 11beta-hydroxysteroid dehydrogenase. Endocrinology. 1999;140(7):3188-96.

31.Bujalska IJ, Walker EA, Hewison M, Stewart PM. A switch in dehydrogenase to reductase activity of 11 beta-hydroxysteroid dehydrogenase type 1 upon differentiation of human omental adipose stromal cells. The Journal of clinical endocrinology and metabolism. 2002;87(3):1205-10.

32.Dominici M, Le Blanc K, Mueller I, Slaper-Cortenbach I, Marini F, Krause D, et al. Minimal criteria for defining multipotent mesenchymal stromal cells. The International Society for Cellular Therapy position 
statement. Cytotherapy. 2006;8(4):315-7.

\section{Figures}
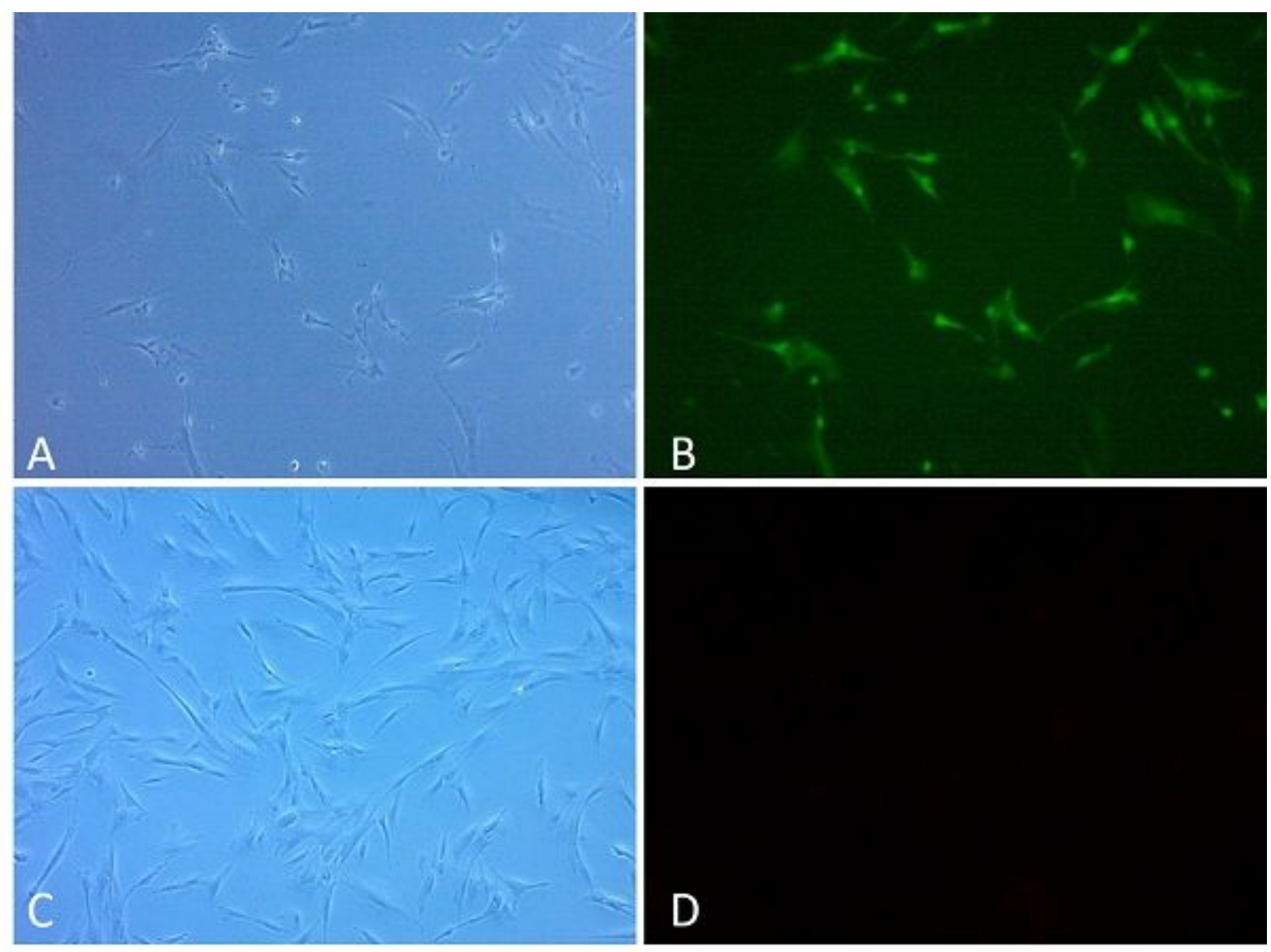

\section{Figure 1}

GFP expression by transgene-activated tMSCs. All images at 200x magnification. (A) Transgene-activated tMSCs imaged using bright field microscopy and (B) fluorescence microscopy showing GFP expression.

(C) Non-transfected MSCs imaged using bright field microscopy and (D) fluorescence microscopy showing lack of GFP expression. 

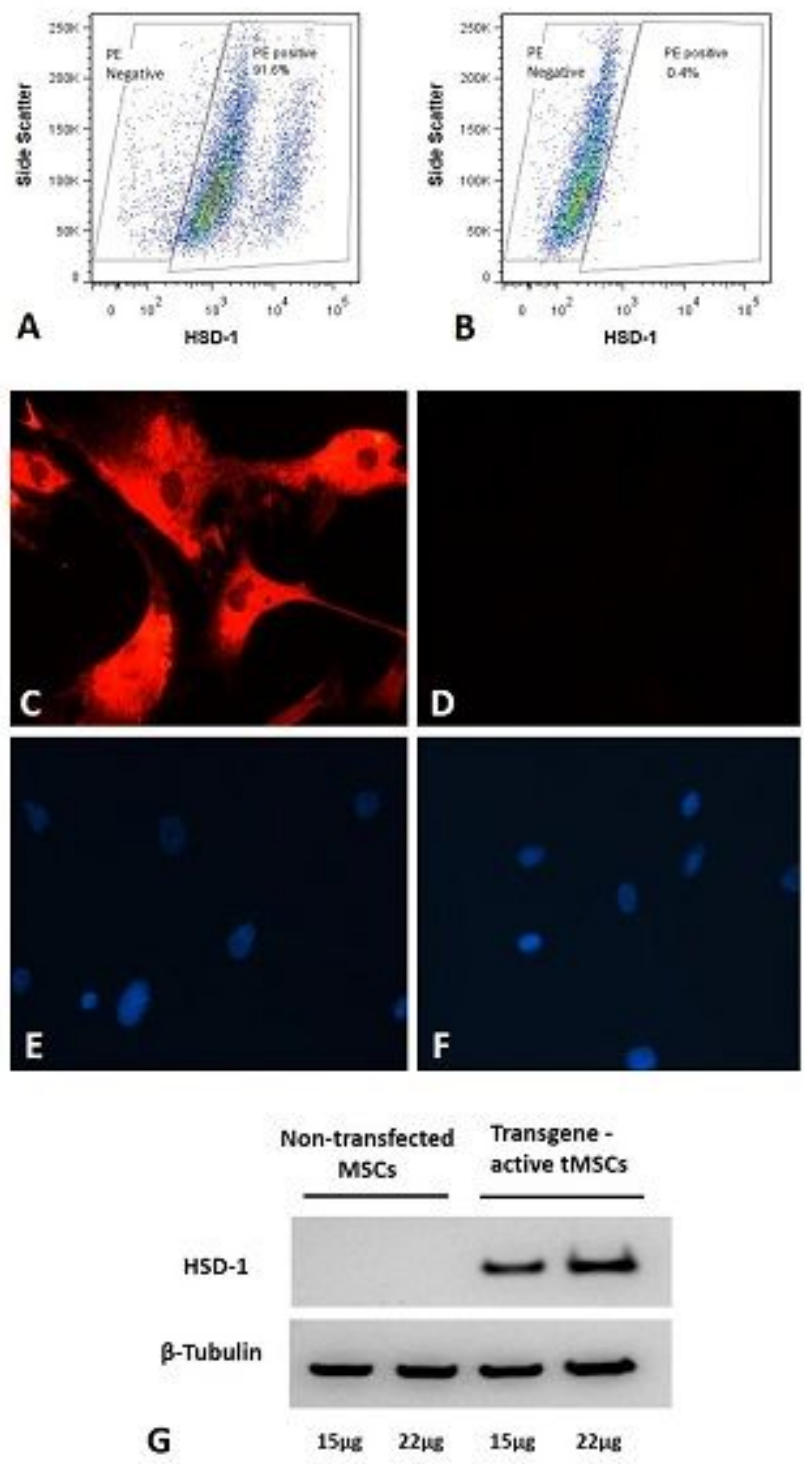

\section{Figure 2}

Assessment of HSD-1 expression in tMSCs. Representative data shown. MSCs and tMSCs were made permeable with saponin buffer prior to intracellular staining for HSD-1. All images at 400x magnification. (A) Representative flow cytometry plot showing HSD-1 staining in transgene-activated tMSCs; data used to calculate transfection efficiency. (B) Flow cytometry plot showing HSD-1 staining in non-transfected MSCs. (C) On fluorescence microscopy using the AF555 filter, transgene-activated tMSCs showed cytoplasmic expression of HSD-1, whereas (D) non-transfected MSCs did not show fluorescence indicating a lack of HSD-1 expression. (E) DAPI nuclear staining of transgene-active tMSCs and (F) nontransfected MSCs shown when viewed under the DAPI filter on fluorescence microscopy. (G) For western blot experiments, $15 \mu \mathrm{g}$ and $22 \mu \mathrm{g}$ protein concentrations were used for each cell type: Image shown is representative of 3 technical replicates. Protein from non-transfected MSCs and transgene-activated tMSCs was labelled for HSD-1; the predicted band size of HSD-1 protein is 38kDa. Transgene-active tMSCs expressed HSD-1 protein whereas non-transfected MSCs did not. The same membrane was 
stripped and re-labelled for beta-tubulin, showing that similar amounts of protein had been loaded for both cell types.

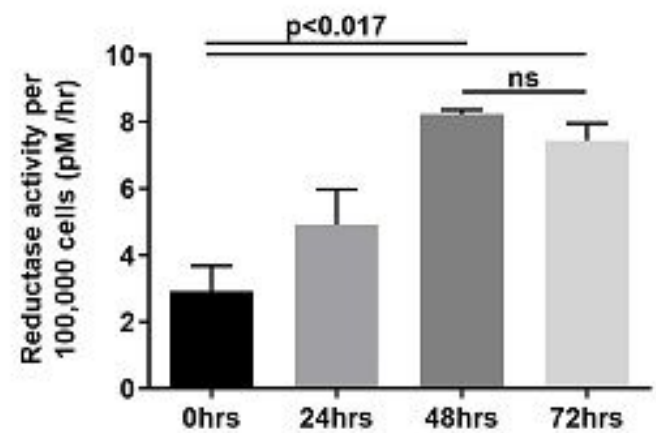

A

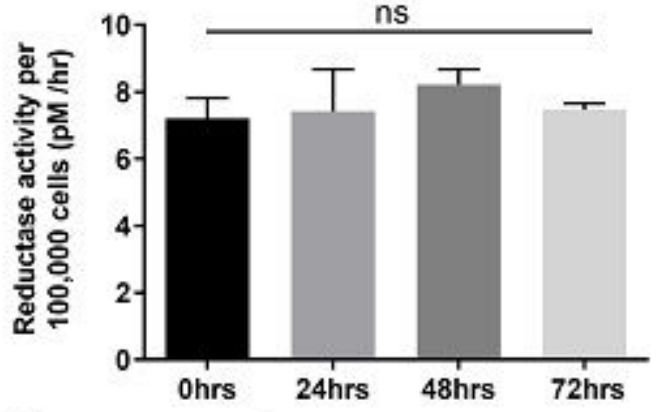

B Duration after doxycycline removal (hrs)
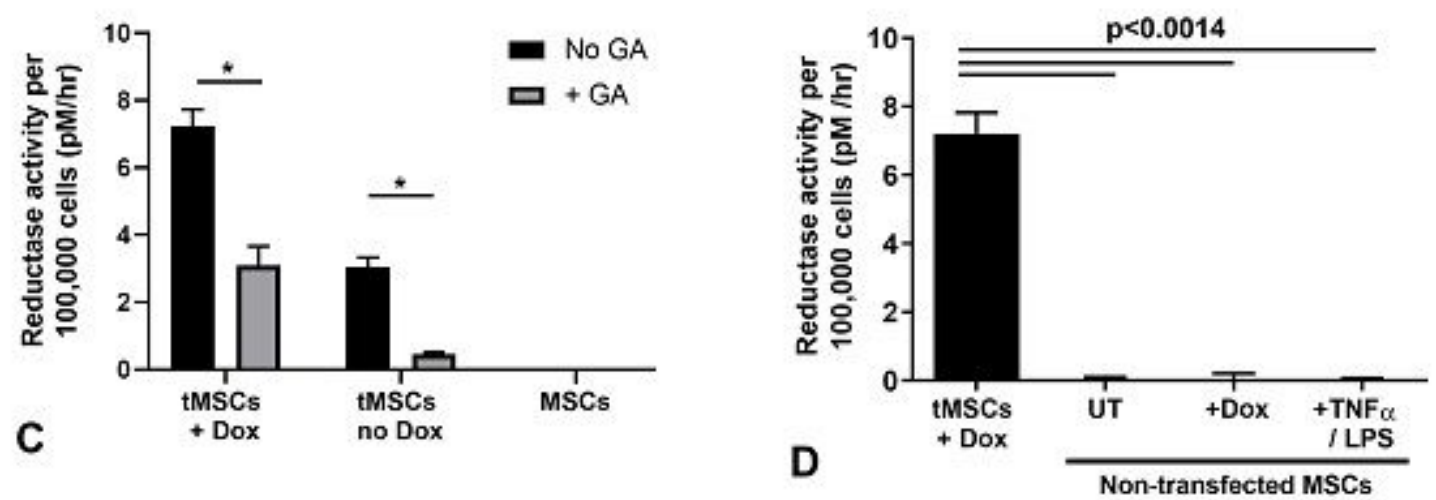

Figure 3

Assessment of HSD-1 functional activity in tMSCs. All data are shown as median and inter-quartile range, with $n \geq 4$ for all conditions. Statistical analysis by Kruskal-Wallis tests and Dunn's multiple comparison test. (A) Reductase activity of HSD-1 tMSCs following differing durations of doxycycline exposure. Following exposure to doxycycline (which acts as a transcriptional activator), tMSCs express HSD-1 with functional reductase activity (Kruskal-Wallis $p=0.001$ ). HSD-1 reductase activity plateaus after 48 hours exposure to doxycycline. There is some baseline HSD-1 reductase activity seen in tMSCs without doxycycline exposure, however this is significantly lower than activity following 48 hours doxycycline treatment (mean rank difference $-11.5, p=0.0025$ ). (B) tMSCs which had previously been exposed to doxycycline for 48 hours, were then cultured in doxycycline-free media for differing durations prior to HSD-1 functional assay. tMSCs retain a constant level of functional HSD-1 reductase activity for 72 hours following doxycycline removal (Kruskal-Wallis $\mathrm{p}=0.303$ ). (C) Incubation with 10-7M glycyrrhetinic acid significantly reduces reductase activity of both doxycycline-treated and untreated tMSCs. MSCs have no detectable HSD-1 reductase activity. GA $=24 \mathrm{hr}$ treatment of $10-7 \mathrm{M}$ glycyrrhetinic acid. Dox $=72 \mathrm{hr}$ treatment of $10 \mu \mathrm{g} / \mathrm{ml}$ doxycycline. Mann-Whitney U test, $\mathrm{n}=4$ for all groups, ${ }^{*} \mathrm{p}<0.05$. (D) HSD-1 reductase activity is negligible in non-transfected MSCs, and exposure to doxycycline or an inflammatory environment (TNFa and LPS) have no effect on MSC HSD-1 activity (Kruskal-Wallis $p<0.0001$ ). 


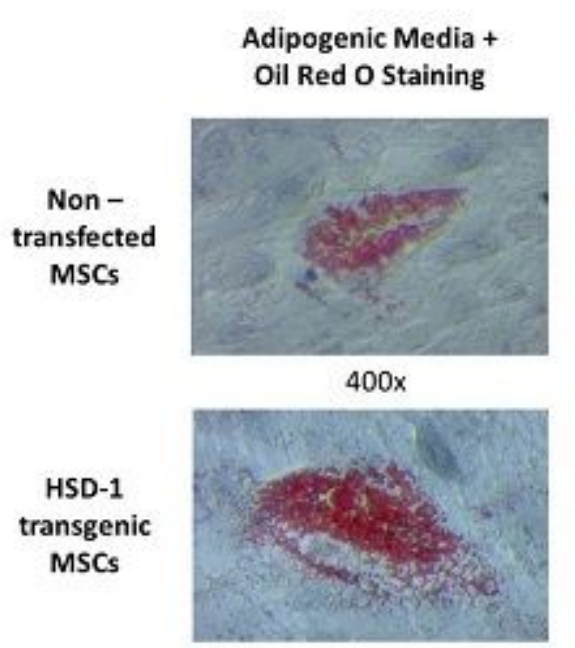

A

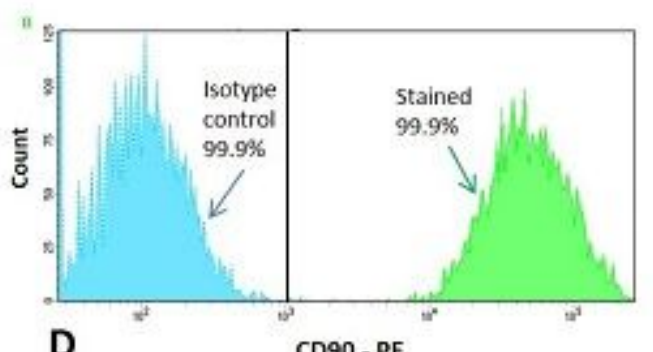

D

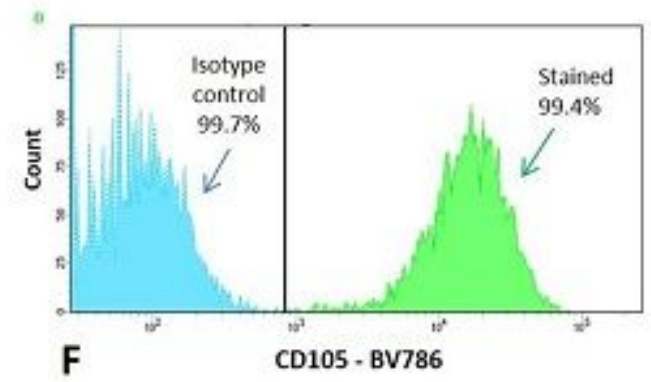

Osteogenic Media +

Alizarin Red S Staining

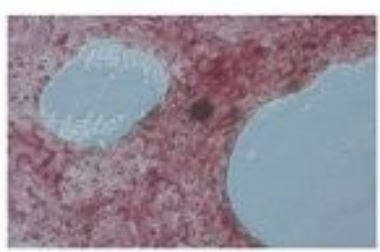

$50 \mathrm{x}$

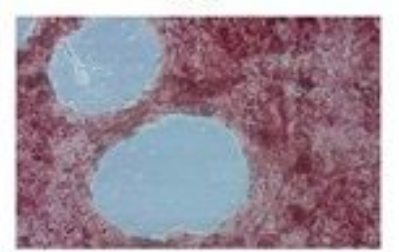

B $\alpha M E M+$

Both Staining

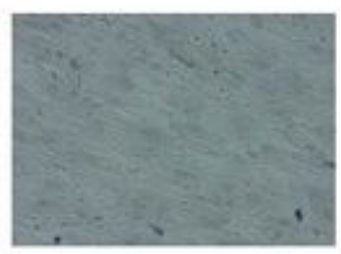

$20 x$

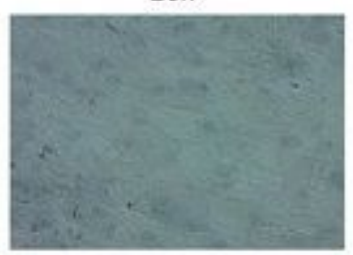

C

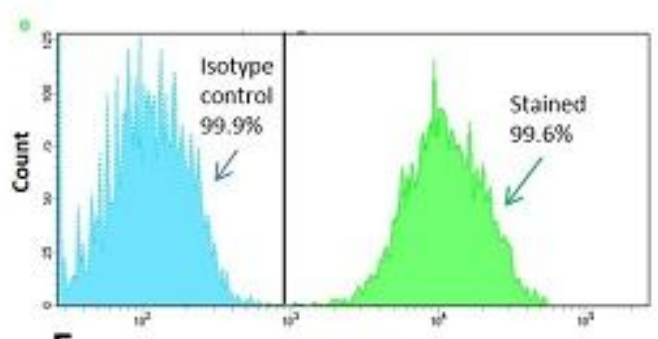

CD73 - BV421

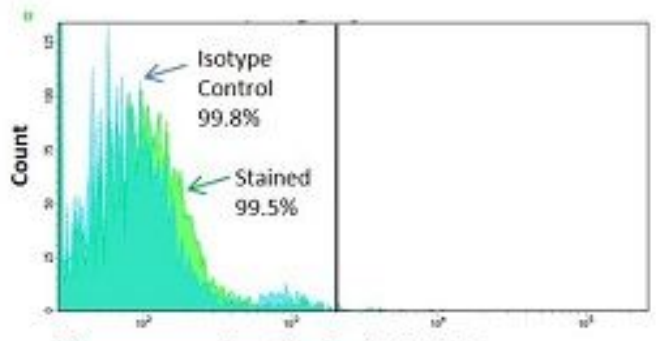

G Negative Cocktall - APC

\section{Figure 4}

Assessment of tMSC phenotype. All cells shown are at passage 4. Non-transfected MSCs and tMSCs were successfully differentiated down (A) adipogenic and (B) osteogenic lineages. (C) When cultured in aMEM including 16\% FBS (control), both MSCs and tMSCs showed no signs of lineage differentiation on dual staining. (D-F) Overlaid fluorescence histograms of HSD-1 tMSCs labelled with antibodies against MSC positive markers (CD90, CD73 and CD105) or isotype controls. Distinct separation of histograms indicate that HSD-1 tMSCs do express surface markers CD90, CD73 and CD105. (G) The negative staining cocktail contained APC-conjugated antibodies against CD14, CD19, CD34, CD45, and HLA-DR. When fluorescence histograms are overlaid, there is near-complete overlap, indicating that HSD-1 tMSCs lack expression of all these surface markers. 